\title{
Zu den syrjänischen Lehnwörtern der obugrischen Sprachen
}

\section{Ostj. kaě pur 'lustig'}

Aus der Mitteilung von Steinitz (OVE 2: 132) kennen wir die folgenden ostjakischen Ausdrücke: Syn. kašli pušli, Kaz.

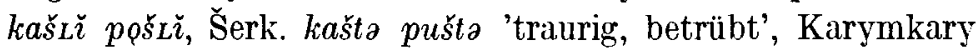
kačč purta 'traurig', kač pur 'lustig', kač pur woj 'lustiges Tier (e. Märchenfigur)'. Vgl. noch (KT 443, 752) Ni. kašpuš, Kaz. kašpoš 'Vergnügen, Annehmlichkeit, Unterhaltung'. Das erste Glied ist mit dem syrjänischen Lehnwort (KT 443) Kaz. kaš 'Vergnügen, Freude; Lust, Wille' usw. (vgl. syrj. gaž 'Freude') identisch (s. Torvones FUF 32: 36). Die Endung -li, - $-i,-t a$ ist am Ende der Form kaš (kačč $<* k a c ̌ t a)$ wie auch der Formen puš, pur ein Karitivbildungssuffix. Das Wort puš, pur ist nur im obigen Ausdruck bekannt. Wenn wir von der logischen und sehr wahrscheinlichen Annahme ausgehen, dass von den Formvarianten die Form pur die ursprünglichere ist und dass das $s$-Element der Formen $p u s ̌, p o \check{s}$ durch den assimilierenden Einfluss des ersten Gliedes (kaš) zu erklären ist, so kann das Wort pur (>puš) auf das folgende syrjänische Wort zurückgeführt werden: (WUo.) V S Peč. Lu. I Ud. P bur 'gut; das Gute'. Die Lautentsprechungen syrj. $b \rightarrow$ ostj. $p$, syrj. $r \rightarrow$ ostj. $r$ sind allgemein üblich. Auch die Substitution von syrj. $u$ durch den ostjakischen Laut $u, Q\left(<\right.$ urostj. $\left.{ }^{*}\right)$ ) ist regelmässig (vgl. Torvonen a.a.0. 128).

Der Umstand, dass das Wörtchen pur, puš selbständig nicht sondern nur in der vorerwähnten Wortverbindung enthalten ist, weist darauf hin, dass der Ausdruck kač pur von einer syrjänischen Konstruktion *bur gaž 'gute Freude, gute Lust' abgeleitet werden kann (vgl. Fokos-Fuchs: aslad bur gǎ́ 'dein freier Wille'); das heisst, das Wort bur ging nicht für sich, 
sondern bloss als Glied des erwähnten Ausdrucks ins Ostjakische über. Die Annahme einer auf Grund des ostj. kač pur zu erwartenden syrjänischen Sequenz gaz bur hat im Syrjänischen gar keine Wahrscheinlichkeit, da das Wortgefüge determiniertes Wort + Attribut dem Geist der syrjänischen Sprache völlig fremd ist. Der Wechsel in der Reihenfolge der Glieder: ${ }^{*} p u r$ $k a c ̌>k a c ̌$ pur 'gute Lust' ging gewiss im Ostjakischen vor sich; dies war dadurch möglich, dass das Wort pur im Ostjakischen keine selbständige Anwendung und Bedeutung hatte.

\section{Ostj. puči: likar p. 'Hinterteil des Schlittens'}

Ostj. (KT 754) Kaz. pøši 'hinterer Teil, Hinterseite (des Menschen, des Tieres); Schwanz', O posi 'Schwanz (des Tieres, Vogels, Fisches)' ist ein syrjänisches Lehnwort: (WUo.) V VU S Peč. I Lu. Le. Ud. P bež 'Schwanz (auch beim Vogel) (V S Lu. Le. P), Schwanz (Peč.), Schwanz (nicht beim Vogel) (Ud.); Schoss am Kleide (V); Hinterteil des Bootes (VU)', (GEN.) PO buz 'Schwanz' (Torvonen FUF 32: 57). Meines Erachtens ist dasselbe syrjänische Wort die Quelle auch des folgenden ostja-

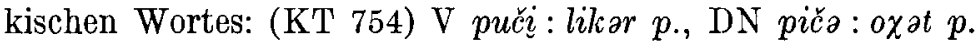
'Hinterteil des Schlittens', Trj. puči 'Schoss, unterer Rand (ringsum) (eines Kleidungsstücks, Oberkleids u.a.)', 'ă $\gamma ә L ~ p$. 'Hinterteil des Schlittens', Kaz. pǔši 'Hinterteil des Schlittens, der Pferdenarte (?) (nicht Rückenbrett); unteres Ende (einer Uferdüne); hintere Spitze (?)', O pusi 'Hinterteil des Schlittens (äusserstes Ende); unteres Ende (einer Uferdüne)'.

Die Lautsubstitution syrj. $b$ - $\rightarrow$ ostj. $p$ - tritt in allen ostjakischen Dialekten einheitlich auf. Im Falle von Kaz. p९ši, 0 posi haben wir es mit einer Lautsubstitution Kaz. $s, \mathrm{O} s \leftarrow$ syrj. $\not$ oder - falls das Wort zu den alten Entlehnungen gehört - mit urostj. $* \varnothing \leftarrow$ syrj. $z$ zu tun. Die inlautende Konsonantenvertretung von V Trj. puči, DN pičə, Kaz. pǔši, O pusi weist eindeutig auf eine Lautsubstitution syrj. $\check{z} \rightarrow$ urostj. ${ }^{*} c ̌ c h i n$. Der Vokal der ersten Silbe der ostjakischen mundartlichen Angaben kann auf drei urostjakische Laute zurückgeleitet werden: *o (Kaz. poši, O posi), *u (V Trj. puči, Kaz. pǔši, O pusi), * $i$ oder *i (DN piča). Die das syrj. $e(<\stackrel{o}{)})$ substituierenden ur- 
ostjakischen Vokale, wie auch die abweichenden Bedeutungen weisen ohne Zweifel darauf hin, dass einerseits Kaz. poš̌, O posi mit der Bedeutung 'hinterer 'Teil, Hinterseite, Schwanz', anderseits V Trj. puči, Kaz. pǔš̌, O pusi mit der Bedeutung 'Hinterteil des Schlittens, Schoss...' im Ostjakischen Entlehnungen aus wahrscheinlich verschiedenen Zeiten, jedoch unbedingt solche von verschiedenen Dialekten sind. DN piča ist wegen seines Lautes $i$ ( $<$ urostj. ${ }^{*} i$ oder $\left.{ }^{*} i\right)$ in der ersten Silbe - trotz der übereinstimmenden Bedeutung - ebenfalls eine von den Formen Trj. puči usw. unabhängige, selbständige dialektale Entlehnung. Für die nichterstsilbigen sekundären Vokale ( $i, i$, , usw.) s. Torvonen a.a.O. 145; Rédei Die syrjänischen Lehnwörter im Wogulischen 52.

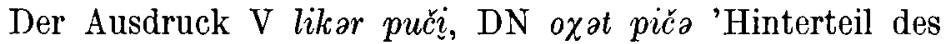
Schlittens' (likar 'Schlitten', oxat ds.) ist wahrscheinlich eine unter dem Einfluss von syrj. (KRSl.) dod'-bež 'задок саней' entstandene hybride Zusammensetzung (blended compound). Kaz. pǔši, O pusi ds. ist entweder eine Rückbildung aus einer früheren Konstruktion * $\chi^{*} \partial L$ pǔš , oder aber die unmittelbare Quelle der Bedeutung 'Hinterteil des Schlittens' - unabhängig von der Zusammensetzung dod-beg - ist das syrjänische Grundwort bež (vgl. VU: 'Hinterteil des Bootes'). Für die Bedeutung ostj. Trj. 'Schoss, unterer Rand (ringsum) (eines Kleidungsstücks, Oberkleids 11.a.)' s. syrj. bęž 'Schoss am Kleide (V)'.

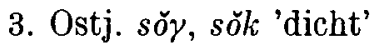

Das im Titel angeführte ostj. (OL 206) V Vj. Trj. sŏ (TEREŠKIN) V sŏk, C saw 'dicht, engmaschig (Netz)' darf trotz der Ähnlichkeit von Form und Bedeutung wegen seines anlautenden $s$ - nicht mit dem Wort (KT 931) Kr. šo $\chi$ 'buschig, dichtbelaubt (Baum)', (PD 2334) Ko. šo $\chi$ 'dicht' etymologisch identifiziert werden. Steinitz (DEWO 253) hält šo für die wahrscheinliche Entlehnung desselben syrjänischen Wortes ček 'dicht', aus dem auch ostj. (KT 931) DN ș̆ək 'Dickicht, Gehölz; dichtbelaubt (Baum)', Kaz. ş̌k 'dichtbelaubt, dicht (Baum, Baumbestand)' usw. stammt (s. Torvonen FUF 32: 79). Die Unregelmässigkeit des an der Stelle des $\ell$ der gebenden Sprache 
auftretenden ostj. o findet im affektiven Charakter des Wortes ihre Erklärung (StEinitz a.a.0.). Das ostj. sŏ $\gamma$, sǒk, saw ist wegen der auffallenden lautlichen und semantischen Übereinstimmung aller Wahrscheinlichkeit nach ebenfalls ein syrjänisches Lehnwort, vgl. syrj. (WUo.) V S Lu. I Ud. P suk'dicht, (v. Flüssigkeiten) dick; Bodensatz', (GEv.) PO suk 'dicht, dickflüssig'. Was die Konsonanten betrifft, bedarf lediglich die Entsprechung syrj. $k \rightarrow$ ostj. $k, \gamma, w$ einer Erklärung (die Lautsubstitution syrj. $s \rightarrow$ ostj. $s$ ist klar). Das syrj. $k$ wurde im Ostjakischen wahrscheinlich als $k$ übernommen, obwohl sporadisch auch die Lautentsprechung syrj. $k \rightarrow$ ostj. $\gamma$ vorkommt (vgl. Torvonen a.a.O. 107ff.). Ostj. $\gamma$ ist durch den Wechsel $k \rightarrow \gamma(>\mathrm{W} w)$ zu erklären. Die Lautsubstitution syrj. $u-$ ostj. $\check{o}\left(<\right.$ urostj. $^{*} \check{o}$ ) ist regelmässig (vgl. Torvonen a.a.O. 128). Über den in der Form $\mathrm{C} s a w$ aufgetretenen Lautwandel * $\check{\gamma} \gamma>$ aw s. STEINitz OstjVok. 125.

\section{Wog. tūr, tōr, ostj. torr 'Schirm der Mütze'}

Im nördlichen Dialektgebiet beider obugrischen Sprachen ist das folgende Wort bekannt: wog. (VNGy. 2: 716) N. tūr, kênt t., tōr 'a sapka prémkarimája v. ernyője; Pelzverbrämung' oder Schirm der Mütze'; ostj. (KT 1011) Kaz. torr 'bunt genähte Mütze aus Fellen (machte ihren Träger unsichtbar)', semtor 'ostj. "silmälasit» (nahasta); ostjakische "Brille» (aus Leder)', O tor 'Schirm, Augenschutz (an der Mütze)'. Aus dem Wogulischen kann auch noch das Element tur, turi der Zusammensetzungen (VNGy. 2: 322, 562) N. kānitur, kānituri 'sapkaernyõ; Mützenschirm; козырек на фурашке', ākätur ds. hierhergestellt werden. Die obugrischen Wörter mögen wegen ihrer begrenzten geographischen Verbreitung und eigenartigen Bedeutung kaum zum genuinen Wortschatz dieser Sprachen gehören. Ich halte es für möglich, dass beide Wörter Entlehnungen von syrj. (WUo.) V VU S Lu. Le. I Ud. P dor 'Rand' sind. Die Konsonantenentsprechung ist evident, so müssen wir sie nicht besprechen. Über die Lautsubstitution syrj. $o \rightarrow$ wog. $\bar{u}\left(<\right.$ urwog. $\left.{ }^{*} \bar{u}\right)$, ostj. $Q$ $\left(<\right.$ urostj. ${ }^{*}$ ) s. RÉdei SLw. 36; ToIvonen FUF 32: 125. Das kurze $u$ des in den Zusammensetzungen kānitur, ẳkätur 
erscheinenden Gliedes -tur hängt wahrscheinlich mit der nichterstsilbigen (unbetonten) Position zusammen. Der $\bar{o}$-Laut $\left(=\bar{\jmath}<\right.$ urwog. $\left.{ }^{*} \bar{a}\right)$ der wogulischen Form tōr kann durch den Lautwechsel urwog. ${ }^{*} \bar{a} \sim{ }^{*} \bar{u}$ erklärt werden. Über den Vokalwechsel in den syrjänischen Lehnwörtern der wogulischen Sprache s. RÉdei SLw. 35, 60. In den obugrischen Sprachen mag sich eine Bedeutungsverengung 'Rand' $\rightarrow$ 'Rand, Krempe, Schirm der Mütze' vollzogen haben.

\section{Ostj. wŭšan 'tanschweise'}

Auf Seite 262 des Wörterbuches KarJaLaInen-Toivonen ist der folgende Ausdruck zu lesen: Ni. wǔšan teta jaśta 'elää läheisessä ystävyydessä, хорошо жить; in enger Freundschaft leben, gut leben' (s. unter dem Stichwort «ǔšan). Die Bedeutungsangabe beruht nicht auf einer wortgetreuen, sondern auf einer sinngemässen Übersetzung aus dem Ostjakischen. Die Bedeutung von zwei Wörtern des aus drei Wörtern bestehenden Ausdrucks (teta, jaśta) ist: 'essen, trinken'. Das Wort wǔšan ist im Wörterbuch nicht selbständig aufgenommen worden, es kommt nur in diesem und in dem nachfolgend zu behandelnden Ausdruck vor. Aus der Kargheit der Belege im Wörterbuch ist darauf zu schliessen, dass sein Anwendungsbereich ziemlich eng und speziell sein mag. Der beschränkte spezielle Gebrauch und die geographische Verbreitung - das Wort kommt nur im Ni.-Dialekt vor - deuten gleicherweise darauf, dass «ǔšan nicht zum genuinen Wortschatz der ostjakischen Sprache gehören kann. Meiner Ansicht nach ist ostj. uŭšan eine Übernahme von syrj. veženn, welches die mit der Instruktivendung versehene Form von syrj. (WIED.) vež 'Kreuz, Quere' (s. noch WUo.: V S Lu. P vež : tuj-v. 'Wegkreuz, Wegscheide') ist. Zur Lösung der Bedeutung von ostj. wǔšan und der wortgetreuen Übersetzung des vorerwähnten Ausdrucks verhelfen uns die folgenden syrjänischen phraseologischen Konstruktionen: (SrSIK) Lu. Skr. Ud. vežen, VO vežen 'в обмен, взамен, в отмену'; (KRSl.) vežen 'взамен, в обмен', $v$. bośtni 1. 'взять, обменив' 2. 'взять, принять в обмен', v. setni 1. 'выдать, обменив', 2. 'вернуть в возмещение', v. loni 
оказаться обмененным, подмененным'. Im Lichte der angeführten syrjänischen Ausdrücke kann die Bedeutung von ostj. wǔšan festgestellt werden: 'tauschweise'. Die wortgetreue Übersetzung der Konstruktion uǔšan teta jaśta lautet: 'tauschweise [d.h. einander abwechselnd, untereinander wechselnd] essen, trinken', folglich 'in enger Freundschaft leben, gut leben'.

Die Lautsubstitutionen syrj. $v \rightarrow$ ostj. $w$, syrj. $\not{z} \rightarrow$ ostj. $\check{s}$, syrj. $n \rightarrow$ ostj. $n$ sind normal. Über dic im syrjänischen Wort vežen anzunehmenden Lautsubstitutionen ursyrj. ${ }^{*} \rightarrow \rightarrow$ urostj. ${ }^{*} i($ nach $w$ Ni. $\check{u})$, syrj. $e(e) \rightarrow$ ostj. $a\left(<\operatorname{urostj} .{ }^{*} \ddot{a}\right)$ s. TorvoNEN FUF 32: 132, 141.

Die syrjänischen Wörterbücher, die ihr Material etymologisch gruppieren (WICнмANN-UotiLs, Foкоs-Fuchs), trennen das syrjänische Substantiv vez 'Kreuz, Quere', (WUo.) $v e z$ : V S Lu. P $t u j-v$. 'Wegkreuz, Wegscheide', (Gex.) PO viž 'Kreuz': tuj- $\imath$. 'Kreuzweg' und das Verb (WUo.) VS Lu. P I Ud. vež- 'wechseln (V S Lu. P I Ud.), tauschen (I), kaufen (I)', (GEN.) PO vi ‘̌z- 'tauschen' voneinander. So verfährt auch das syrjänische etymologische Wörterbuch (ESK). Doch ist diese Trennung nicht berechtigt, da die Glieder des substantivisch-verbalen Wortpaars sowohl rom lautlichen, als auch vom semantischen Gesichtspunkt unbedingt zusammengehören. Vgl. wotj. (Muxk.) S vož 'Ort, wo zwei Flüsse od. Wege zusammenfliessen; Flussmündung, Wegscheide', (Wiснм.) G vož : śures $v$. 'Wegscheide' $\sim$ (MuNk.) S K vošt- 'tauschen, um-

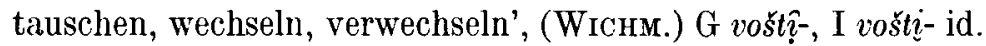
Der zu erschliessende urpermische Vokal der ersten Silbe dürfte ${ }^{*} e$ gewesen sein (s. E. Itkonen FUF 31: 311) ${ }^{1}$. Der

${ }^{1}$ Es ist überraschend, dass die PO Dialektform von syrj. veštti- 'loskaufen, auslösen, einlösen, befreien, erlösen' (PO vesšti-, GEN., LYTK.), das als kausative Ableitung von syrj. vez- 'wechseln, tauschen' betrachtet werden muss, auf urpermisches ${ }^{*} e$ deutet (s. E. ItKonen a.a.O.; ESK). Es ist sehr wahrscheinlich, dass das $e$ von PO ve št $t$ - durch den $e$-Laut des homonymen veršti- 'hastig bewegen' ( V S Lu. P. vešti'wegrücken [tr.], bewegen') beeinflusst wurde. Das ESK stellt das Verb veští- 'loskaufen, auslösen . . ' zur Familie von vešjị- 'wegrücken (intr.)', vešt $i$ - 'wegrücken (tr.)'. Das stösst aber auf grosse semantische Schwierigkeiten. 
Grund des Zusammenhangs der Bedeutungen von vež 'Kreuz, Quere' und vež- 'tauschen, wechseln' mag die Anschauung sein, dass die Personen, die den Tausch abwickeln, ihre Tauschobjekte - in überwiegenden Fällen - etwa q u e r dahinstreckend einander ïberreichen. ${ }^{1}$

6. Ostj. «ǔšan 'kreuzweise, krumm, schief'

Der andere Beispielsatz des Wörterbuches KaRJALAIneNToIvonen, in welchem ostj. wǔšan vorkommt, lautet folgen-

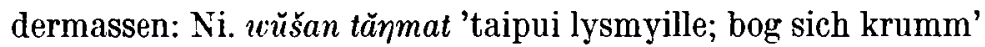
(262). Die Bedeutung von tăクmat ist: '[er, sie] ging hinein, ist hineingegangen', vgl. Ni. tă - - 'irgendwo hineingehen' (KT 1069). Die Bedeutung 'tauschweise' des vorher behandelten Wortes wǔšan passt nicht zu diesem Ausdruck. Wenn wir aber voraussetzen, dass syrj. vežen auch in der Bedeutung 'kreuzweise' ins Ostjakische überging (vgl. KRSl. vežęn 'крестом, крест-накрест', s. noch Fokos-Fuchs SrSlK unter pad-vez), können wir dem Ausdruck wǔšan tăłmat die Bedeutung '[er, sie] ging quer hinein, ist quer hineingegangen' beimessen. Und wenn wir sogar in Betracht ziehen, dass die Bedeutung des ostjakischen Wortes eventuell auch durch die mit der Lativendung -iń, -iń versehene Form von syrj. vez 'Kreuz, Quere' (Fokos-Fuchs: V vežin, vežin, Le. vežin 'krumm [V], schief [Le.]') beeinflusst wurde, kann die Bedeutung des ostjakischen Ausdrucks noch genauer erklärt werden: '[er, sie] ging schief hinein, ist schief hineingegangen'.

7. Wog. visar, ostj. wusar 'Strudel'

Wog. (ČRn.) N. visar 'небольшой водоворот', ostj. (K'T 262) Ni. Kaz. wǔšar, O uusär 'in Wirbeln dahinfliessender Strom (Ni. Kaz., O), Strudel (0)' < syrj. (SrSlK) Vm. vežer 'омут'. Die Lautsubstitutionen syrj. $v \rightarrow$ wog. $v(=w)$, ostj. $w$, syrj.

${ }^{1}$ Die Gleichung des syrjänischen Verbs vež- 'tauschen, wechseln' mit dem finnischen Wort vaihta- (ESK mit Fragezeichen) bedarf vom lautlichen Gesichtspunkt - vielleicht FP *wajšs- > urpermisch *vež- einer weiteren Untersuchung. 
$\check{z} \rightarrow$ wog. $s$, ostj. $\check{s}, s$, syrj. $r \rightarrow$ wog.-ostj. $r$ sind regelmässig. Auch die Lautsubstitution syrj. $e \rightarrow$ wog. $i$ ( $i$ oder $i$ ), ostj. Ni. Kaz. $\check{u}, \mathrm{O} u$ (< urostj. $\left.{ }^{*} i\right)$ ist regelmässig. Syrj. $e \rightarrow$ urostj. ${ }^{*} i$ deutet darauf hin, dass der ursyrjänische Vokal ein * $e$ gewesen war. Das ursyrj. ${ }^{*} e$ wird im Ostjakischen nämlich durch urostj. ${ }^{*} e$ substituiert (vgl. Torvonen FUF 32: $129 \mathrm{ff}$.). Über die Lautsubstitution syrj. $e \rightarrow$ wog. $a$, ostj. $a, a$ s. RÉDEI SLw 50; Torvones a.a.O. 141. Die Bedeutungen der syrjänischen und obugrischen Wörter decken sich genau.

KÁROLY RÉdEI 